\title{
The Relationship between Sacral Kyphosis and Pelvic Incidence
}

\author{
George McKay, Peter Alexander Torrie, Georgina Dempster, Wendy Bertram, Ian Harding \\ Department of Spinal Surgery, Southmead Hospital, Bristol, UK
}

\begin{abstract}
Study Design: Retrospective cohort study.
Purpose: Evaluate the fixed anatomical parameter of sacral kyphosis (SK) and its relationship with pelvic incidence (PI).

Overview of Literature: Pelvic parameters determine pelvic and lumbar spinal position. Studies have defined normative values, and have evaluated the role of these parameters in clinical practice. It has been suggested that a ratio of sacral slope (SS)/PI $<0.5$ predisposes to spinal pathology. PI=SS+pelvic tilt (PT) and therefore for a given PI, patients with a higher SS due to an elevated SK will potentially predispose to an unfavourable SS/PI ratio.

Methods: CT measurements of SS and PI were made in 100 consecutive patients from our database. Imagings without clear landmarks were excluded. PI and SK were measured using standardised techniques. Pearson's correlation was used to assess association between PI and SK, in addition to the correlation between age and the pelvic parameters. Gender specific values for PI and SK were compared using an unpaired Student $t$-test.

Results: Ninety-five patients (52 females) with a mean age 51.3 years were available for analysis. A strong positive correlation between the PI and the SK was identified (Pearson's coefficient $=0.636, R^{2}$ value $=0.404$ ). Neither PI nor SK had a statistically significant correlation with age $(p=0.721$ and $p=0.572$, respectively). The mean values of both the $\mathrm{Pl}$ and SK were statistically significantly lower in females when compared to males ( $p=0.0461$ and $p=0.0031$, respectively).

Conclusions: A strong correlation between PI and SK exists and is a reflection of different pelvic morphologies. SK partially determines SS and a relatively high SK compared to PI will result in less ability to change PT and a potentially unfavourable SS/PI ratio, which could theoretically contribute to clinical pathology.
\end{abstract}

Keywords: Sacrum; Pelvis; Deformity; Spine; Anatomy

\section{Introduction}

The importance of sagittal spinopelvic parameters is increasingly well recognised as being an important tool in assessing the spine, planning surgery, and affecting outcome [1-6]. Initially, there was an emphasis on the C7 plumb line or sagittal vertical axis but as our understanding has evolved, different concepts have developed. This includes a more global approach to alignment analysis, with a better understanding of compensatory mechanisms during dynamic balance of the body, in addition to the static alignment in stance. Authors have described various

Received Nov 30, 2016; Revised Apr 13, 2017; Accepted Apr 22, 2017

Corresponding author: George McKay

Department of Spinal Surgery, Southmead Hospital, Dorian Way, Westbury-on-Trym, Bristol, BS10 5NB United Kingdom

Tel: +44-1179-505050, E-mail: mr.g.mckay@googlemail.com 
tilt angles [7], different lumbar morphologies [8], and ideal pelvic tilt (PT) angles/equations $[9,10]$ that have sought to refine our understanding of static alignment further. There is much debate about how these parameters can or should be interpreted but the concept of pelvic incidence (PI) being a fixed anatomical parameter [11] is universal, although it may differ between sex $[12,13]$ and race [1418]. It is accepted that the pelvis can roll from an anteverted to a retroverted position to posturally accommodate as required, limited by hip anatomy and maximum hip extension. It is also recognised that patients with higher PI have a greater propensity to do so than those with a lower incidence, i.e., they have a larger range of pelvic motion to potentially accommodate thoracolumbar pathology if it exists [19].

It is well known that sacral slope (SS) and PT are reciprocal and that when one goes up the other must come down to equal the PI. However, these numbers as an isolated entity do not necessarily relate to the clinical situation. It is worth considering that when PI is low, patients may only be able to minimally accommodate for this with a small amount of variation in tilt, and vice versa. It may therefore be more realistic to consider ratios of values compared to the fixed anatomical parameter of incidence rather than the absolute values [12,20-22]. Recently consideration has been given to the concept of an optimal SS to PT ratio with normal values of this described [12]. Abnormal ratios have been seen in flatback deformity [20], and potential clinical application of these ratios has been described with regards to spondylolisthesis classification and management $[21,22]$. It has been postulated that a $\mathrm{PT} / \mathrm{PI}$ ratio of greater than 0.5 , or equally an SS/PI ratio of less than 0.5 , may predispose to spinal problems [12]. An inability to attain this ratio may therefore result in the development of problems or an inability to compensate for thoracolumbar pathologies. Any contribution to a relatively higher SS compared to PT for a given PI may therefore be important. A high sacral kyphosis (SK) could result in a higher SS for a given PI, leading to the inability of the patient to attain the ideal PT/SS ratio or even potentially allow the patient to be able to tilt the pelvis more posteriorly to allow better compensation. We therefore hypothesize that SK could be an important parameter when considering the sagittal plane.

SK has been defined as the angle between a line joining the midpoint of the superior and inferior borders of S1 and the line joining the inferior borders of S2 and S4 [23].
SK, like PI, is a fixed anatomical parameter and a reflection of pelvic morphology. We hypothesize that SK is proportional to PI, and furthermore that patients who have a proportionately high SK and thus a potentially high SS may also have a high PI to facilitate a favorable PT/PI and SS/PI ratio facilitating pelvic compensation for thoracolumbar malalignment. The aim of this study is to measure and assess these parameters.

\section{Materials and Methods}

We analysed the computed tomography (CT) lumbar spine scans of 100 consecutive existing patients from our unit's spinal database (Southmead Hospital, Bristol, UK), who had been previously investigated with full length lumbar spine CT scans including a lateral scanogram of femoral heads and sacrum as demonstrated in Fig. 1. The indications for these scans were varied and included: unexplained pain, investigation of symptoms in patients not suitable for MRI scanning, possible deformity/spondylolysis/spondylolisthesis, trauma/possible fracture, amongst others. Each scan was therefore clinically indicated and performed for reasons pre-dating this study, and as such it was not necessary to gain approval from our Institutions Regulatory Board or ethics committee with regards to this or any aspect of our study design.

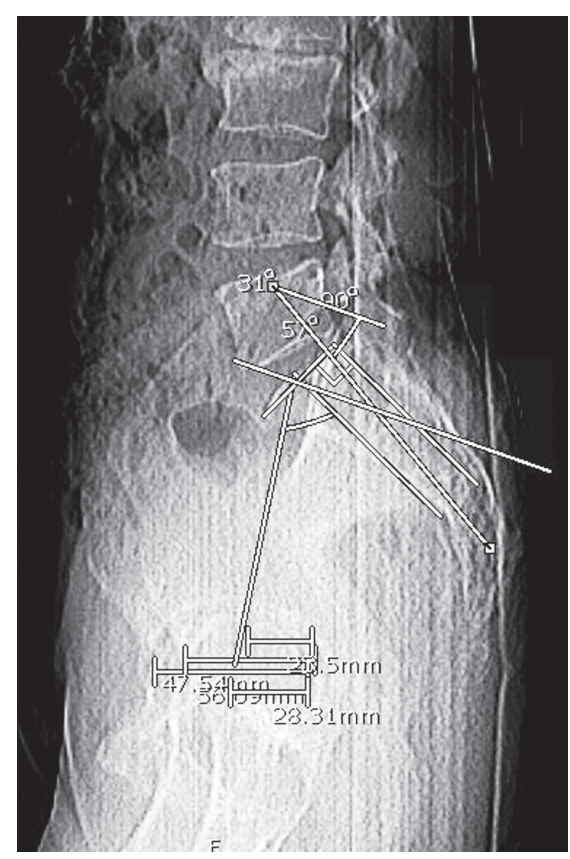

Fig. 1. Representation of computed tomography scanogram used to measure parameters. 
Inclusion criteria required that patients were over 18 years of age and had a completed CT lumbar spine series with a lateral scanogram available for review on our hospital PACS (Patient Archiving Computer System). Patients were excluded from the study if they were not over 18 years of age. Patients with any previous instrumented surgery to the lumbosacral area or pelvis were excluded from the study, as were those with unclear bony landmarks on the scanogram.

Study data was collected by two authors and interobserver reliability was compared with the senior author using Cronbach's alpha. SK was measured using the technique SK was measured using the technique utilised by Wang et al. [23]. This defines SK as the angle subtended between the perpendicular line transecting the midpoint between the anterior and posterior borders of the superior endplate of $\mathrm{S} 1$ and the line transecting the midpoint between the anterior and posterior borders of the inferior endplate of S2-S4 (Fig. 2). The PI was measured from the sagittal plane CT scanograms.

Statistical analysis was conducted using IBM SPSS ver. 19.0 (IBM Corp., Armonk, NY, USA). Pearson's correlation was used to determine whether a linear relationship between PI and SK was present, and to assess the pelvic parameters' correlation with age. An unpaired two-tailed Student $t$-test was used to compare gender specific difference for PI and SK. Statistical significance was accepted at a $p$-value $<0.05$.

\section{Results}

Five patients were excluded from the study as they did not meet inclusion criteria: two patients were under 18 years of age and three scanograms did not have clear bony landmarks. Ninety-five patients (52 female and 43 male) with a mean age 51.3 years (range, $18-85$ years) were included in the study. The measurements of both pelvic parameters,

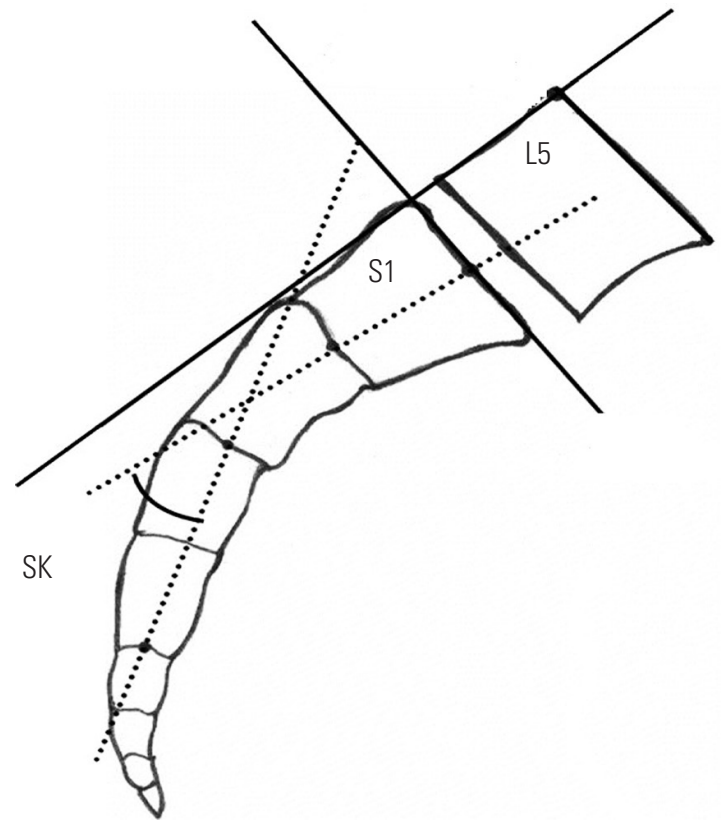

Fig. 2. Graphical representation of measurement of SK, sacral kyphosis.

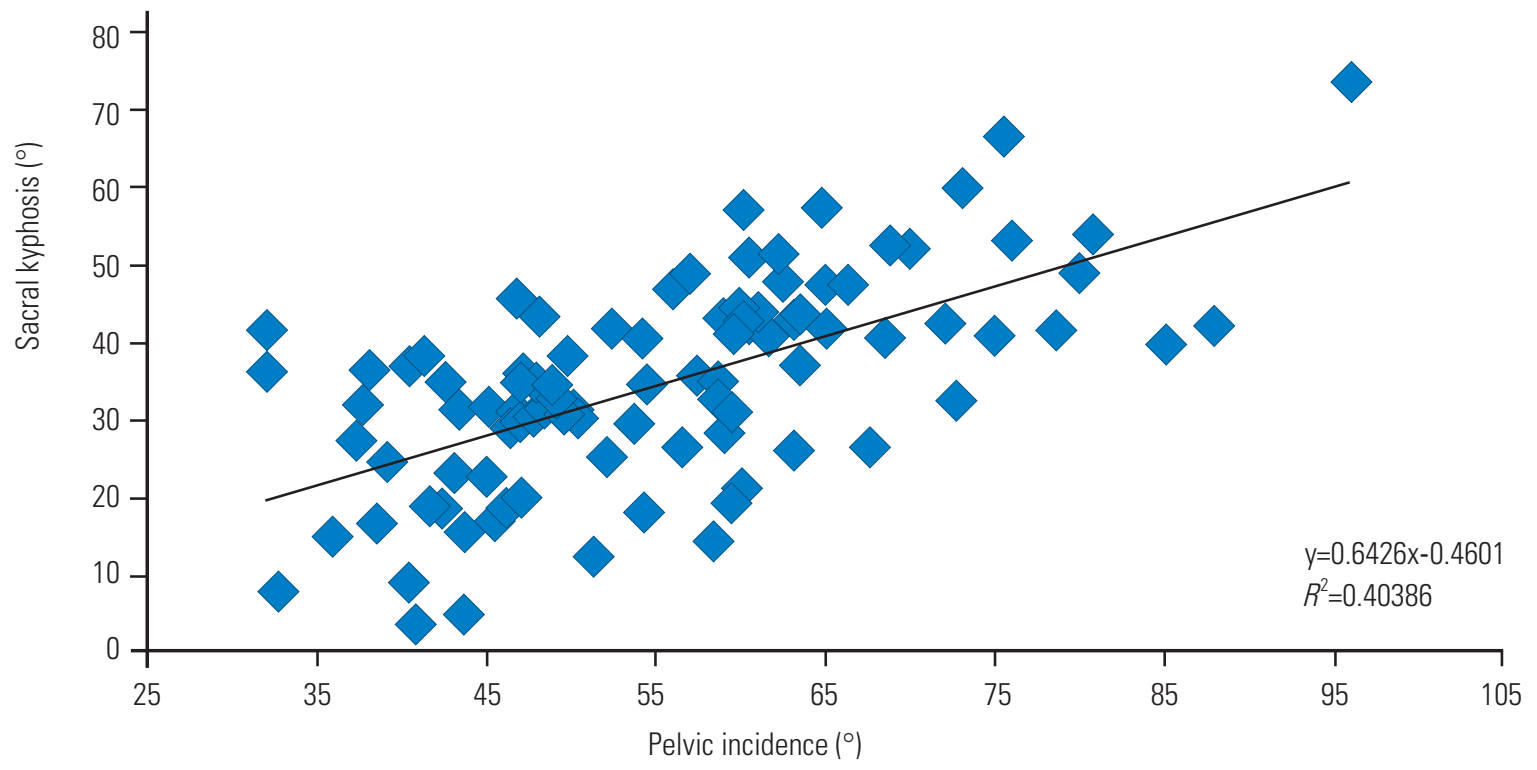

Fig. 3. Correlation between pelvic incidence and sacral kyphosis. 
PI and SK, in all 95 remaining patients were recorded. Excellent inter-observer internal consistency was observed for both PI and SK ( $\alpha=0.985$ and 0.991, respectively).

Both pelvic parameters (PI and SK) followed parametric distributions with mean values of $55.3^{\circ}$ (range, 52.6-57.9) and $35.1^{\circ}$ (range, 32.4-37.8), respectively. A highly statistically significant positive correlation between the PI and SK was identified (Pearson's $R=0.636, R^{2}=0.404$ and $p<0.0001$ ) (Fig. 3). Neither PI nor SK had a strong correlation or reached statistical significance when correlated with age (Pearson's $R=0.037, p=0.721$ and Pearson's $R=0.057, p=0.572$, respectively). The mean ratio of the SK to the PI was 0.635 (standard deviation $[S D]=0.208$ ), with a \pm 2 SD range of SK/PI values being $0.219-1.051$.

The mean values of PI (52.9 and $58.2^{\circ}$, respectively) and SK $\left(31.5^{\circ}\right.$ and $39.4^{\circ}$, respectively) were lower in females when compared to males. The two-tailed unpaired Student $t$-test identified gender specific variations in the observed values for both PI and SK ( $p=0.0461$ and $p=0.0031$, respectively). Both subgroups (males and females) showed a statistically significant positive correlation between the PI and SK in both males (Pearson's $R=0.78, p<0.0001$ ) and females (Pearson's $R=0.410, p=0.0025$ ).

\section{Discussion}

The relationship between SS and PI is well established $[24,25]$ as is the notion that certain pathologic processes are associated with a high PI [23,26-29]. Less is known however about the relationship between the more global anatomy of the sacrum and its relationship to PI.

Our results have shown a significant positive correlation between SK and PI, in that a high SK is correlated with a high PI. In anatomic terms we can rationalise that a high SS is associated with a higher degree of SK for a given PI, and that therefore results in a greater potential range for PT to accommodate compared to the situation where SK is low for the same incidence in a different individual. Our data supports previous work showing no significant correlation between PI and age [24], supporting the premise that PI is a fixed anatomic parameter over time. This was also true for the correlation between SK and age supporting $\mathrm{SK}$ as a fixed anatomic parameter within the pelvis.

The fact that there is a close correlation between PI and SK means that the anatomical PI angle is reflected in sacral anatomy in individuals which allows patients with a higher PI to roll the pelvis in the sagittal plane through a wider range of motion. We can also assume that the pelvis is able to compensate for this kyphotic morphology by increasing the value of PT and therefore increasing the capacity to position the pelvis through a wider spectrum of angles. This has obvious implications, as limitations in this respect would impair the ability of the spine as a whole to maintain an economic sagittal balance in the presence of pathologic processes that alter the sagittal profile. In other words, if a patient does have a high SK they are also likely to have a relatively high PI and therefore the ratios of SS/ PI are favourably maintained, as suggested by Mac-Thiong et al. [12]. This compensation cannot occur if the patient has a high SK with a low PI and it is possible that in this situation problems may occur when there is a mismatch in these pelvic morphological parameters. This could be a particular problem with outliers where there is a mismatched PI to SK e.g., SK is high and PI relatively low or vice versa.

Our data set contains two subgroups of very different outliers, highlighted in Fig. 3. One group have a high PI with relatively low SK (low SK/PI ratio). If we therefore attribute such patients as having a potentially low SS and examine their SS/PI ratio, this is much smaller than the 0.5 value suggested by Mac-Thiong et al. [12] as potentially predisposing certain individuals to spinal pathology. At the other end of the spectrum we also have a group, which have a low PI and high SK (high SK/PI ratio). In this scenario we have a potentially low PT/PI ratio. This cohort represents the group of patients with potentially the most significant inability to compensate for sagittal imbalance. This may too have implications with regard to the rehabilitation of patients suffering from spinal pathology.

This study is intended as a preliminary anatomical study and has limitations. Selection bias exists due to our cohort comprising of patients who have been referred to the spine service with pathology of varying natures. We sought to limit this by excluding patients with sacral dome moulding, spondylolisthesis, spondylolysis, and instrumentation, but otherwise taking a consecutive series of our patients. The lack of clinical data is a limitation but we emphasise that this is a theoretical hypothesis suggesting the premise that there was always a relationship between SK and PI. We have shown that in most patients the two pelvic parameters positively correlate, but not in all and we suggest that the outliers in whom correlation does not exist and there is mismatch may either develop spinal pathology as a result or more likely, are unable to 
facilitate the necessary pelvic compensations to balance in stance. It is now necessary to investigate this with regard to pathologies in a clinical setting.

\section{Conclusions}

This study is the first to support a positive correlation between PI and SK. There is an anatomic association between SS and SK and this work supports the concept that as morphology of the sacrum changes so does the potential range of pelvic movement in the sagittal plane. This could potentially lead to unfavourable numeric ratios between pelvic position (SS/PT) and pelvic anatomy (PI), with theoretical impact upon clinical pathology. Further clinical evaluation of our findings is required to further delineate this relationship.

\section{Conflict of Interest}

No potential conflict of interest relevant to this article was reported.

\section{References}

1. Glassman SD, Berven S, Bridwell K, Horton W, Dimar JR. Correlation of radiographic parameters and clinical symptoms in adult scoliosis. Spine (Phila $\mathrm{Pa}$ 1976) 2005;30:682-8.

2. Glassman SD, Bridwell K, Dimar JR, Horton W, Berven S, Schwab F. The impact of positive sagittal balance in adult spinal deformity. Spine (Phila Pa 1976) 2005;30:2024-9.

3. Maruo K, Ha Y, Inoue S, et al. Predictive factors for proximal junctional kyphosis in long fusions to the sacrum in adult spinal deformity. Spine (Phila $\mathrm{Pa}$ 1976) 2013;38:E1469-76.

4. Smith MW, Annis P, Lawrence BD, Daubs MD, Brodke DS. Early proximal junctional failure in patients with preoperative sagittal imbalance. Evid Based Spine Care J 2013;4:163-4.

5. Annis P, Lawrence BD, Spiker WR, et al. Predictive factors for acute proximal junctional failure after adult deformity surgery with upper instrumented vertebrae in the thoracolumbar spine. Evid Based Spine Care J 2014;5:160-2.

6. Park SJ, Lee CS, Chung SS, Lee JY, Kang SS, Park SH. Different risk factors of proximal junctional kypho- sis and proximal junctional failure following long instrumented fusion to the sacrum for adult spinal deformity: survivorship analysis of 160 patients. Neurosurgery 2017;80:279-86.

7. Schwab FJ, Smith VA, Biserni M, Gamez L, Farcy JP, Pagala M. Adult scoliosis: a quantitative radiographic and clinical analysis. Spine (Phila $\mathrm{Pa} 1976$ ) 2002;27:387-92.

8. Roussouly P, Gollogly S, Berthonnaud E, Dimnet J. Classification of the normal variation in the sagittal alignment of the human lumbar spine and pelvis in the standing position. Spine (Phila $\mathrm{Pa} 1976$ ) 2005;30:346-53.

9. Schwab F, el-Fegoun AB, Gamez L, Goodman H, Farcy JP. A lumbar classification of scoliosis in the adult patient: preliminary approach. Spine (Phila Pa 1976) 2005;30:1670-3.

10. Bess S, Schwab F, Lafage V, Shaffrey CI, Ames CP. Classifications for adult spinal deformity and use of the Scoliosis Research Society-Schwab Adult Spinal Deformity Classification. Neurosurg Clin N Am 2013;24:185-93.

11. Janssen MM, Drevelle X, Humbert L, Skalli W, Castelein RM. Differences in male and female spinopelvic alignment in asymptomatic young adults: a three-dimensional analysis using upright lowdose digital biplanar X-rays. Spine (Phila Pa 1976) 2009;34:E826-32.

12. Mac-Thiong JM, Roussouly P, Berthonnaud E, Guigui P. Age- and sex-related variations in sagittal sacropelvic morphology and balance in asymptomatic adults. Eur Spine J 2011;20 Suppl 5:572-7.

13. Vialle R, Levassor N, Rillardon L, Templier A, Skalli W, Guigui P. Radiographic analysis of the sagittal alignment and balance of the spine in asymptomatic subjects. J Bone Joint Surg Am 2005;87:260-7.

14. Banno T, Togawa D, Arima $\mathrm{H}$, et al. The cohort study for the determination of reference values for spinopelvic parameters (T1 pelvic angle and global tilt) in elderly volunteers. Eur Spine J 2016;25:3687-93.

15. Zhu Z, Xu L, Zhu F, Jiang L, et al. Sagittal alignment of spine and pelvis in asymptomatic adults: norms in Chinese populations. Spine (Phila Pa 1976) 2014;39:E1-6.

16. Zarate-Kalfopulos B, Romero-Vargas S, OteroCamara E, Correa VC, Reyes-Sanchez A. Differences in pelvic parameters among Mexican, Caucasian, and 
Asian populations. J Neurosurg Spine 2012;16:516-9.

17. Lee CS, Chung SS, Kang KC, Park SJ, Shin SK. Normal patterns of sagittal alignment of the spine in young adults radiological analysis in a Korean population. Spine (Phila Pa 1976) 2011;36:E1648-54.

18. Duval-Beaupere G, Marty C, Barthel F, et al. Sagittal profile of the spine prominent part of the pelvis. Stud Health Technol Inform 2002;88:47-64.

19. Harding IJ. Understanding sagittal balance with a clinical perspective. Eur J Phys Rehabil Med 2009;45:571-82.

20. Gottfried ON, Daubs MD, Patel AA, Dailey AT, Brodke DS. Spinopelvic parameters in postfusion flatback deformity patients. Spine J 2009;9:639-47.

21. Labelle H, Mac-Thiong JM, Roussouly P. Spinopelvic sagittal balance of spondylolisthesis: a review and classification. Eur Spine J 2011;20 Suppl 5:641-6.

22. Mac-Thiong JM, Wang Z, de Guise JA, Labelle H. Postural model of sagittal spino-pelvic alignment and its relevance for lumbosacral developmental spondylolisthesis. Spine (Phila Pa 1976) 2008;33:2316-25.

23. Wang Z, Parent S, Mac-Thiong JM, Petit Y, Labelle $H$. Influence of sacral morphology in developmental spondylolisthesis. Spine (Phila Pa 1976) 2008;33:2185-91.
24. Mac-Thiong JM, Berthonnaud E, Dimar JR 2nd, Betz RR, Labelle H. Sagittal alignment of the spine and pelvis during growth. Spine (Phila $\mathrm{Pa} 1976$ ) 2004;29:1642-7.

25. Legaye J, Duval-Beaupere G, Hecquet J, Marty C. Pelvic incidence: a fundamental pelvic parameter for three-dimensional regulation of spinal sagittal curves. Eur Spine J 1998;7:99-103.

26. Labelle H, Roussouly P, Berthonnaud E, et al. Spondylolisthesis, pelvic incidence, and spinopelvic balance: a correlation study. Spine (Phila Pa 1976) 2004;29:2049-54.

27. Antoniades SB, Hammerberg KW, DeWald RL. Sagittal plane configuration of the sacrum in spondylolisthesis. Spine (Phila Pa 1976) 2000;25:1085-91.

28. Hanson DS, Bridwell KH, Rhee JM, Lenke LG. Correlation of pelvic incidence with low- and high-grade isthmic spondylolisthesis. Spine (Phila Pa 1976) 2002;27:2026-9.

29. Jentzsch T, Geiger J, Bouaicha S, Slankamenac K, Nguyen-Kim TD, Werner CM. Increased pelvic incidence may lead to arthritis and sagittal orientation of the facet joints at the lower lumbar spine. BMC Med Imaging 2013;13:34. 